\title{
Design and Microfabrication of Cooling Arm for Inertial Confinement Fusion Application
}

\author{
Bin $\mathrm{Xu}^{1,2}$, Jing-quan Liu ${ }^{1, *}$, Shui-dong Jiang ${ }^{1}$, Gang Tang ${ }^{1,2}$, \\ Xiao-xiao Yan ${ }^{1,2}$, Bin Yang ${ }^{1}$, Xiang Chen ${ }^{1}$ and Chun-sheng Yang ${ }^{1}$ \\ ${ }^{1}$ National Key Laboratory of Science and Technology on Micro/Nano Fabrication, \\ Department of Micro/Nano Electronics, Shanghai Jiao Tong University, \\ 800 Dong Chuon Road, Shanghai 200240, China \\ ${ }_{2}^{2}$ Jiangxi Province Key Laboratory of Precision Drive and Control, \\ Department of Mechanical and Electrical Engineering, Nanchang Institute of Technology, \\ 289 Tian Xiang Road, Nanchang 330099, China
}

(Received May 21, 2015; accepted August 20, 2015)

Key words: cooling arm, inertial confinement fusion, MEMS, temperature simulation, optimization design

Inertial confinement fusion (ICF) mainly uses strong focus laser beams to irradiate the target to obtain sufficient energy. The ignition target is an essential factor for inducing a fusion reaction within a capsule. The cooling arm that connects the cooling source to the hohlraum is an important part of the ignition target, which is used to obtain an accurate and uniform temperature field for the deuterium-tritium ice pellet. To improve the temperature uniformity of the connecting surfaces between the cooling arm and the hohlraum, the cooling arm is designed to have a groove structure. On the basis of theoretical analysis and temperature simulation, the three-branch cooling arm was found to have a smaller temperature difference on the clamping surface and better mechanical strength than the cooling arm with fewer branches. Moreover, the fracture failure test shows that the fracture force of the three-branch cooling arm is $1.12 \mathrm{~N}$, which is larger than that of the two-branch cooling arm. Because the smoothness of the connecting surface between the cooling arm and the hohlraum has a considerable effect on heat transfer, a $\mathrm{KOH}$ :isopropanol (IPA) solution is used to polish the rough deep reactive ion etching (DRIE) surface.

\section{Introduction}

Inertial confinement fusion (ICF) is an approach to fusion that relies on the inertia of the fuel mass to provide confinement. ${ }^{(1-3)}$ The ignition target is an essential factor for inducing a fusion reaction within a capsule. The target mainly contains a thermal mechanical package (TMP) and a capsule (depicted in Fig. 1). ${ }^{(4,5)}$ The support cooling arm terminates at an aluminum can, a part of the thermal mechanical package, which ${ }^{*}$ Corresponding author: e-mail: jqliu@sjtu.edu.cn 


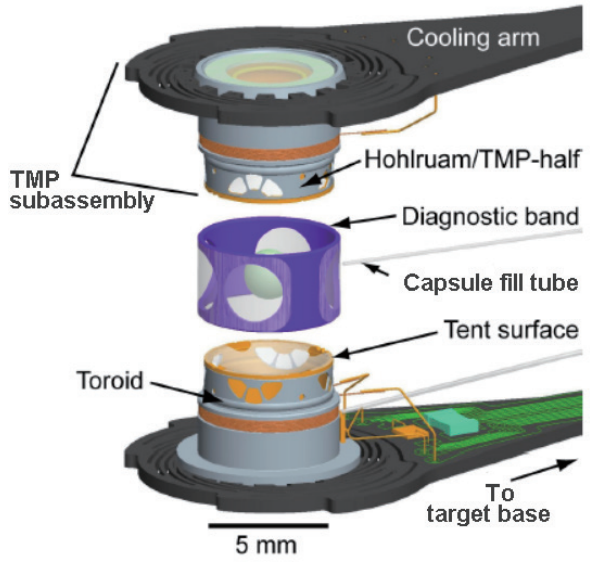

Fig. 1. (Color online) Model of ICF ignition target (exploded).

holds the target hohlraum. The final ends of the support cooling arms must grip the hohraum can in a precise configuration to maintain the temperature of the hohlraum at $18.3 \mathrm{~K}$, and the temperature differences across the target must be less than $0.5 \mathrm{mK}$. This can be achieved by using an intricate design at the joint between the support arm and the hohlraum, which guides the heat path symmetrically in a ring around the joint. ${ }^{(6-8)}$

Therefore, the cooling arm at the target plays an important role in thermal conduction and positioning. For the thermal conduction, it is necessary for the cooling arm to have sufficient contact with the hohlraum, so the clamping claws at the end of the cooling arm should have a high vertical sidewall and a smooth surface. The deep reactive ion etching (DRIE) process can etch a very complex structure, but the etched sidewall has many scallops, and it is difficult to keep the sidewall vertical when etching deep grooves. Huda et al. ${ }^{(9,10)}$ achieved a smooth sidewall surface by optimizing the etch/passivation ratio of the DRIE process. To further improve the surface smoothness, $\mathrm{KOH}$ :isopropanol (IPA) etching can be used to remove the etch scallops caused by DRIE. ${ }^{(1-14)}$

Here, we propose a novel three-branch structure cooling arm prepared by a microfabrication process, which has a small temperature difference and a large deformation tolerance. In order to improve the heat transfer from the cooling arm to the hohlraum, the smoothness of the clamping surface is improved by KOH:IPA polishing, which can highly increase the contact area.

\section{Design of Cooling Arm}

As a key part of the ignition target assembly, the cooling arm is used to transfer heat, maintain the required temperature, and position the hohlraum. As the cooling arm works at $18.3 \mathrm{~K}$, the material should have high thermal conductivity at cryogenic temperature and could also be fabricated by microfabrication technology. As a commonly used semiconductor material, (110) silicon is selected to fabricate a cooling arm owing to its high thermal conductivity, Young's modulus, and resistivity features. 
The cooling arm contains a positioning ring that consists of several clamping claws, as shown in Fig. 2. The clamping head located on one side of the cooling arm was designed to achieve both positioning and heat transfer. As the silicon material was chosen, the good thermal conduction between the cooling arm and the TMP assembly depends on the contact area. It requires that the clamping surfaces of the cooling arm have a uniform curvature with the hohlraum. In order to obtain large contact areas between them, a cooling arm with the largest number of clamping claws is needed. Figure 2 shows the schematic diagram of the assembly of the TMP. It is more difficult to assemble the hohlraum to the cooling arm when the number of clamping claws increases. Considering the maximum number of clamping claws and the difficulty of assembly, the cooling arm structure is designed with 16 clamping claws, as shown in Fig. 5.

In the temperature control process, the cooling arm is cooled by connecting with a cooling source at the tail end of the arm, so the temperature of the clamping claws is below the target temperature at the beginning. By using a heater, the temperature of the claws can reach the target temperature of $18.3 \mathrm{~K}$. The heat transmission from the heater to each claw is shown Fig. 3(a). The length of the heat transfer path of the claws at area 1 is shorter than that of the claws at area 3, so the temperature difference among these claws is large. In order to lessen the difference in heat transfer length to each claw, a groove is added in the clamping head, which we name as a one-branch structure, as shown in Fig. 3(b). Points 1 and 2 in the clamping ring are a pair of isothermal points, as the distance from the heater to these two points is the same. The heat transfers to every claw through these two isothermal points, the difference in heat transfer path length is smaller than the cooling arm length with no groove, and the temperature difference among all the claws will be reduced. To further reduce the temperature difference of every claw, as shown in Fig. 3(c), another grade of grooves is added to the clamping head of the cooling arm. The numbers of isothermal points becomes four around the clamping ring, so the temperature difference of every claw can be further reduced. Restricted by the space of the clamping head of the cooling arm, only three grades of grooves, a three-branch structure, can be added, and eight isothermal points can be distributed around the clamping ring, as shown in Fig. 3(d), making the temperature difference smaller. Through optimized designing, the structure is finally established, as shown in Fig. 4. In addition, the branch structure can reduce the rigidity because some material in the clamping head is removed. Thus, this structure of the cooling arm has a small elastic modulus, and the stress can be reduced while clamping the hohlraum in the same deformation.

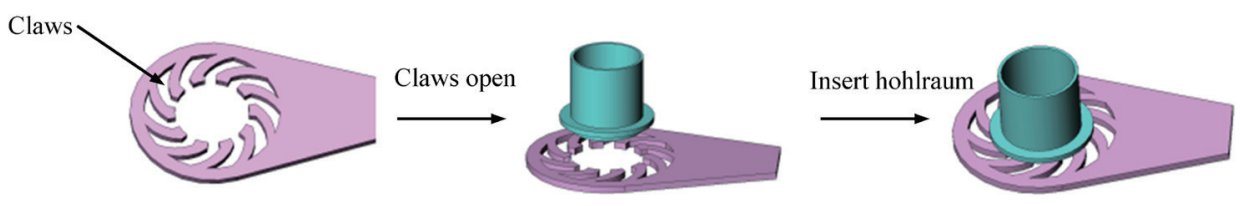

Fig. 2. (Color online) Schematic diagram of TMP assembly. 


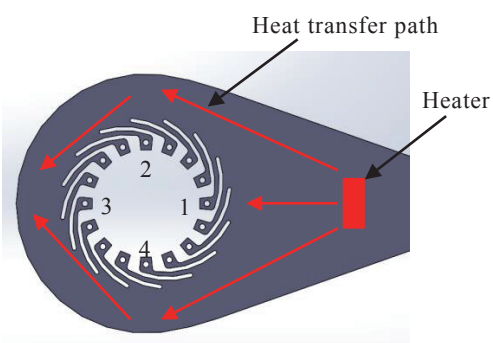

(a)

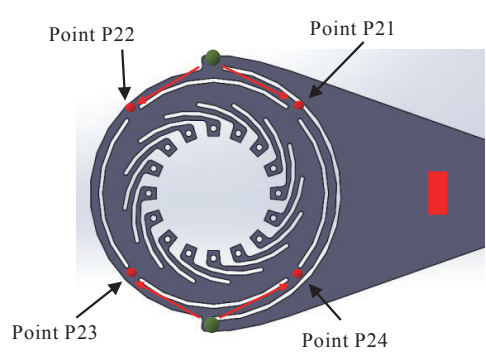

(c)

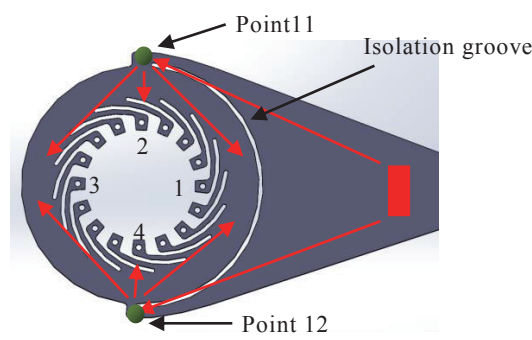

(b)

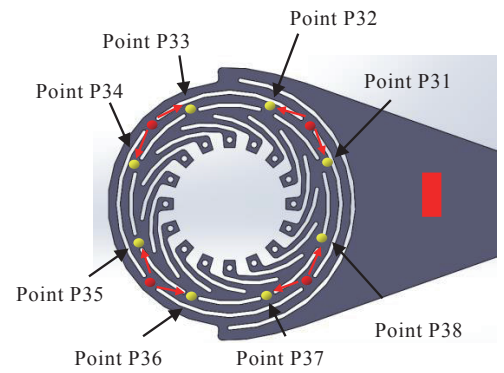

(d)

Fig. 3. (Color online) Head structure of cooling arm. (a) No-branch cooling arm. (b) Onebranch cooling arm. (c) Two-branch cooling arm. (d) Three-branch cooling arm.

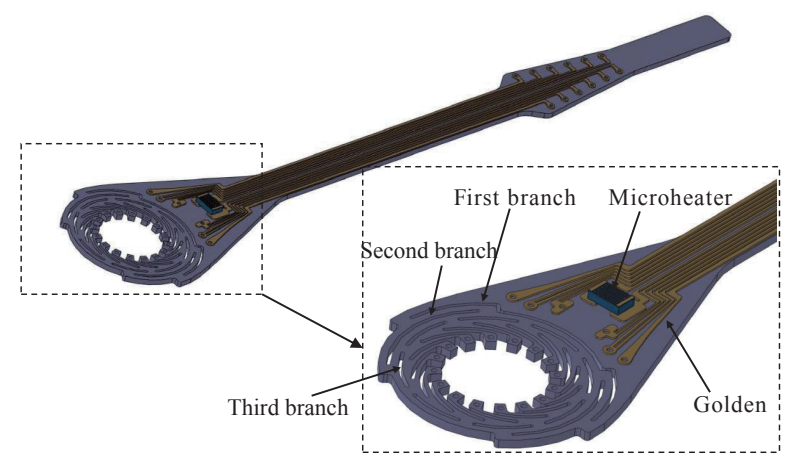

Fig. 4. (Color online) Cooling arm with three branches.

The analysis software COMSOL is used to calculate the temperature difference of the clamping surface and the strength of the cooling arms, and the simulation result of the three-branch cooling arm is shown in Fig. 5(a). In order to calculate the temperature difference of each type of clamping arm, the temperature of the center point on the clamping surface of every clamping arm is plotted in Fig. 5(b). The temperature 


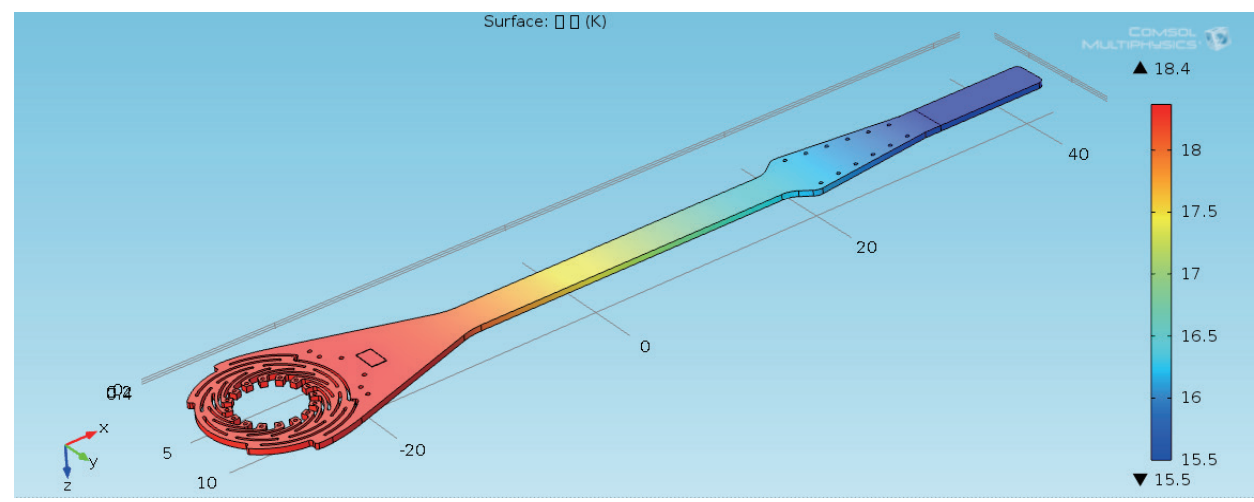

(a)

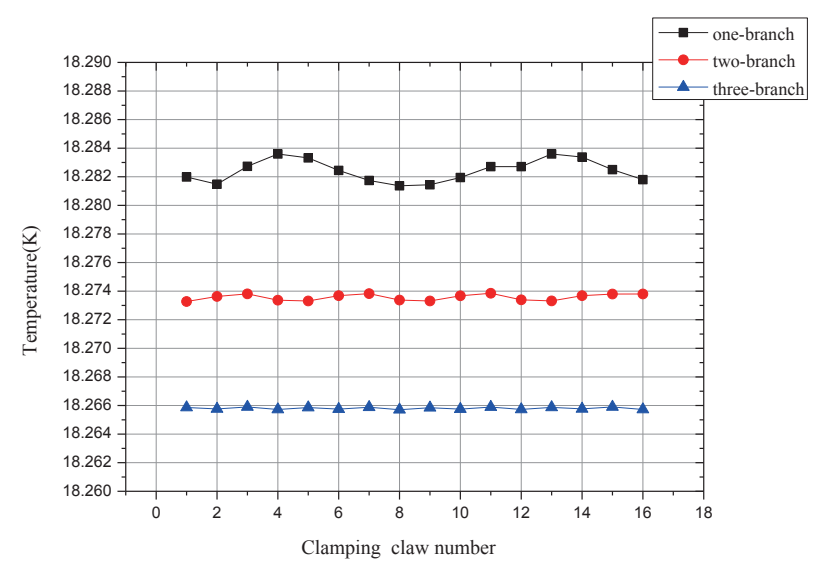

(b)

Fig. 5. (Color online) Temperature simulation of cooling arm. (a) Temperature distribution of three-branch cooling arm. (b) Temperature of every clamping surface of the cooling arms with different groove branches.

difference of the three-branch cooling arm is $0.19 \mathrm{mK}$, which meets the temperature requirement (less than $0.5 \mathrm{mK}$ ). As can be seen from Fig. 5(b), the cooling arm with fewer branches has a larger temperature difference. The temperature differences of the two-branch and one-branch cooling arms are 0.58 and $2.22 \mathrm{mK}$, respectively.

While clamping the TMP assembly, clamping arms will deform $20 \mu \mathrm{m}$ in the normal direction against the clamping surface. The software COMSOL is used to analyze the stress distribution of the cooling arms, as shown in Fig. 6. The maximum stress of the cooling arm with three grades is $0.499 \mathrm{GPa}$. The cooling arms with fewer groove branches have a higher maximum stress. Thus, the three-branch cooling arm has better mechanical strength. 


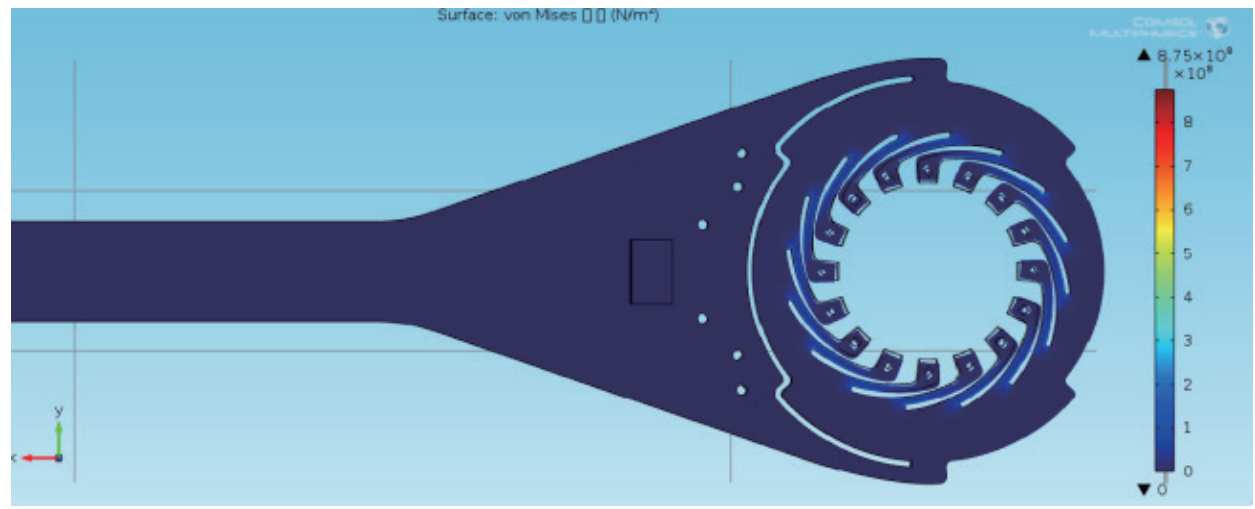

(a)

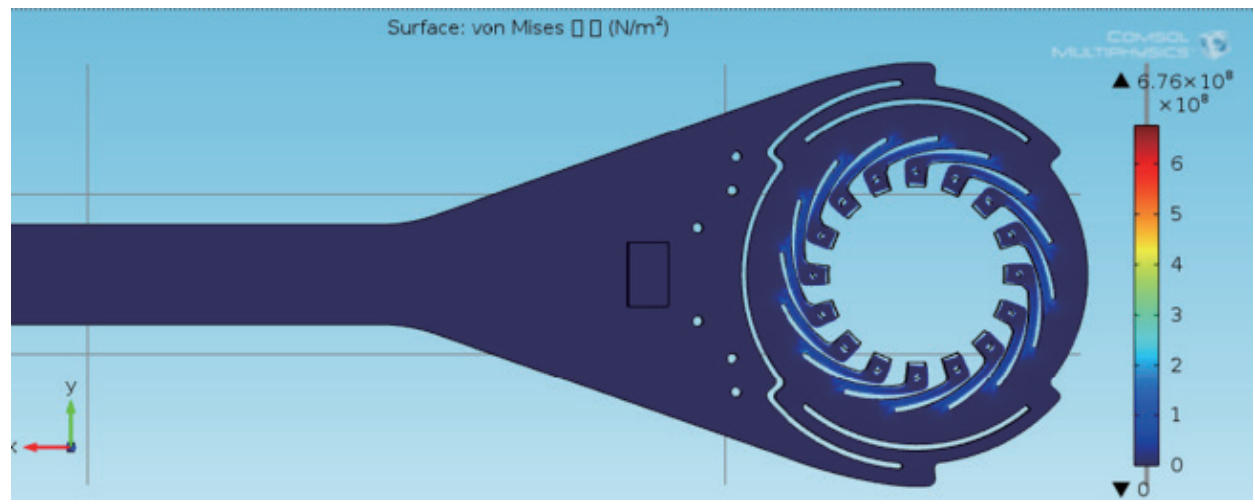

(b)

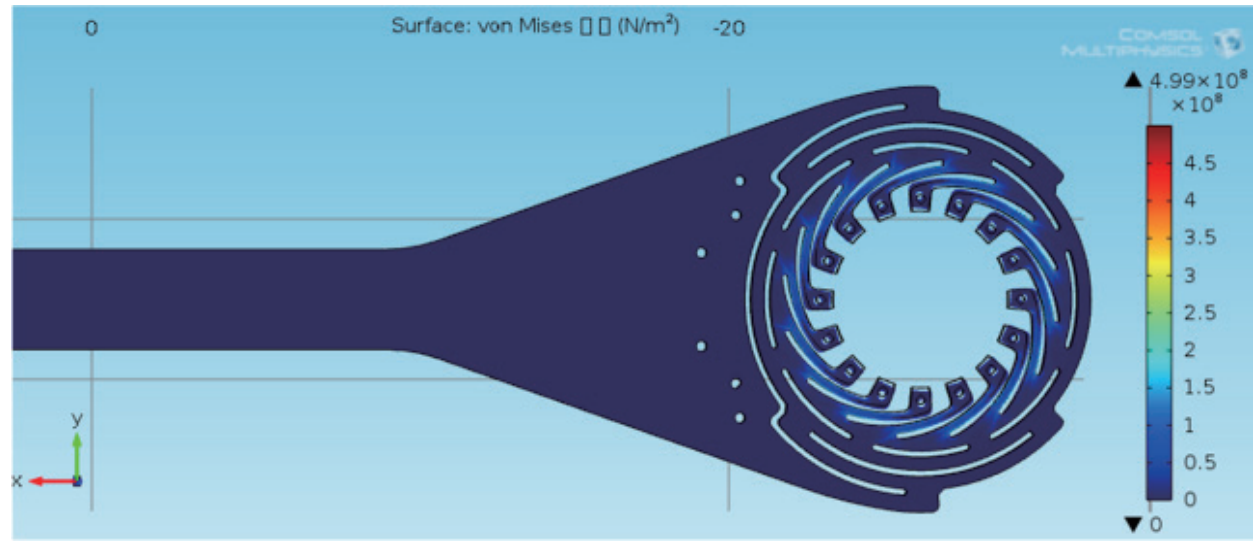

(c)

Fig. 6. (Color online) Stress distribution of cooling arm with the radial displacement of $20 \mu \mathrm{m}$. (a) Stress distribution of one-branch cooling arm. (b) Stress distribution of two-branch cooling arm. (c) Stress distribution of three-branch cooling arm. 


\section{Fabrication of Cooling Arm}

The microfabrication of the cooling arm is shown in Fig. 7. In step (1), the oxidized (110) silicon wafer was cleaned, baked, and coated with an adhesive layer (HMDS). In step (2), the $\mathrm{SiO}_{2}$ on one side of the silicon wafer is removed by reactive ion etching (RIE). Then, in step (3), approximately $1.5 \mu \mathrm{m}$ of a thin photoresist was spin-coated on the substrate and the photoresist was prebaked on the hot plate. After that, in step (4), the wafer was exposed by using a contact mask aligner with a ultraviolet (UV) light source, developed, and then subjected to postbaking. In step (5), the $\mathrm{SiO}_{2}$ layer where the temperature sensor and heater were placed was etched off by RIE, because the thermal conductivity of $\mathrm{SiO}_{2}$ is much lower than that of silicon, which will lower the heat transfer efficiency. In step (6), the wafer was ultrasonically cleaned with acetone and IPA for five minutes. Then, steps (3) and (4) were repeated in steps (7) and (8), respectively. In step (9), the $\mathrm{Cr} / \mathrm{Au}(50 / 150 \mathrm{~nm})$ layers were sputtered on top of the $\mathrm{SiO}_{2}$ layer as the electric layer. After that, a lift-off process was carried out in step (10), and the $\mathrm{Cr} / \mathrm{Au}$ on the photoresist was removed. In step (11), an approximately $20-\mu \mathrm{m}$-thick photoresist (AZ4620) was coated on the wafer and prebaked on the hot plate. In step (12), the photoresist was exposed, developed, and subjected to postbaking. In step (13), the $\mathrm{SiO}_{2}$ layer was patterned for the subsequent DRIE by RIE. In step (14), through-wafer DRIE

(1)

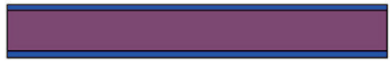

(2)

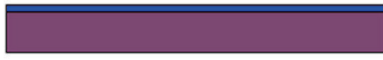

(3)

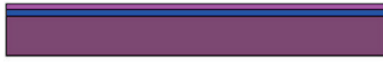

(4)

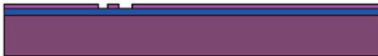

(5)

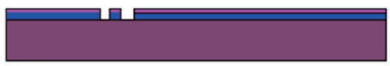

(6)

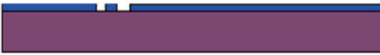

(7)

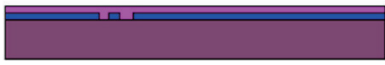

(8)

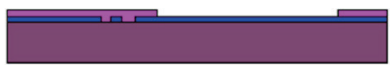

(9)

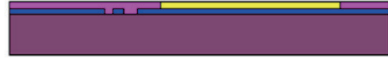

(10)

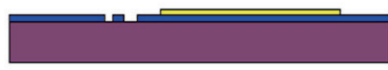

(11)

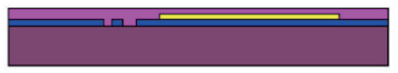

(12)

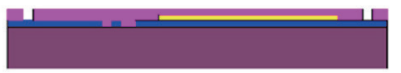

(13)

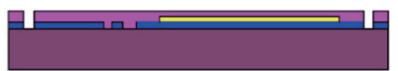

(14)

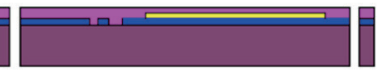

(15)

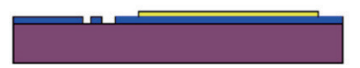

$\square \mathrm{Ni} / \mathrm{Au} \square \mathrm{SiO}_{2} \square \mathrm{Si} \square \mathrm{PR}$

Fig. 7. (Color online) Fabrication process: (1) wafer cleaning, (2) etching off the $\mathrm{SO}_{2}$ layer by RIE, (3) photoresist spin-coating, (4) lithography, (5) etching off $\mathrm{SO}_{2}$ layer by RIE, (6) wafer cleaning, (7) repeat step (3), (8) repeat step (4), (9) sputtering $\mathrm{Cr} / \mathrm{Au}(50 / 150 \mathrm{~nm}$ ) layers, (10) lift-off, (11) 20 $\mu \mathrm{m}$ photoresist (AZ4620) coating, (12) lithography, (13) etching off the $\mathrm{SO}_{2}$ layer by RIE, and (14) etching through the wafer by DRIE. 
was completed. The parameters of the inductively coupled plasma (ICP) etching, which are listed in Table 1, were optimized to obtain the vertical sidewall. In the last step, the phororesist is removed with acetone and IPA.

The fabricated cooling arm is shown in Fig. 8. A scanning electron microscopy (SEM) image is taken to measure the key dimensions of the cooling arm, as shown in Figs. 8(b) and $8(\mathrm{c})$. The diameter of the clamping surface is $5.483 \mathrm{~mm}$, which is only $3 \mu \mathrm{m}$ larger than the design dimension, and the verticality of the clamping sidewall is $89.9^{\circ}$.

The DRIE sidewalls of the silicon wafer are rough because of the scalloping effect, as shown in Fig. 9(a). Because the smoothness of the connecting surface between the cooling arm and the hohlraum has a considerable effect on heat transfer, KOH:IPA was used to remove the scallops and striation roughness that the DRIE process left. Because the rate of $\mathrm{KOH}$ etching silicon is lowest along the (111) plane, the clamping face of the cooling arm can achieve a vertical and smooth surface. As can be seen from the atomic force microscopy (AFM) test, the sidewall surface morphology was highly improved after KOH:IPA polishing, as shown in Fig. 9(b).

Table 1

Parameters of ICP.

\begin{tabular}{lcccc}
\hline Circle & $\mathrm{SF}_{6}$ flow & $\mathrm{C}_{4} \mathrm{~F}_{8}$ flow & ICP power & RF power \\
\hline Etch time $(8 \mathrm{~s})$ & $450 \mathrm{sccm}$ & 0 & $2300 \mathrm{~W}$ & $50 \mathrm{~W}$ \\
Passivation time $(3 \mathrm{~s})$ & 0 & $190 \mathrm{sccm}$ & $1650 \mathrm{~W}$ & $20 \mathrm{~W}$ \\
\hline
\end{tabular}

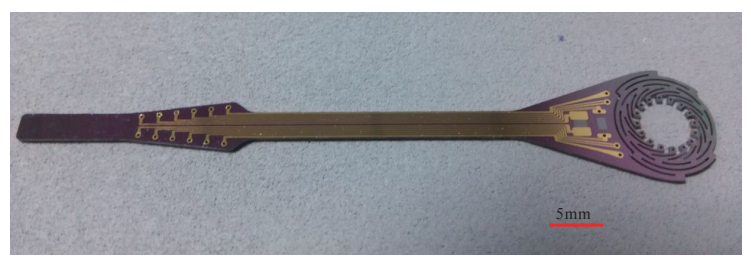

(a)

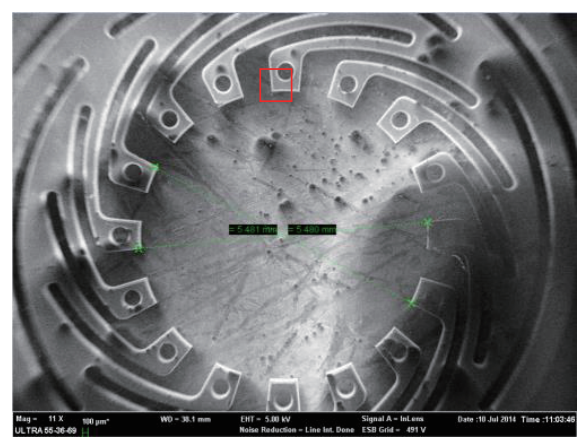

(b)

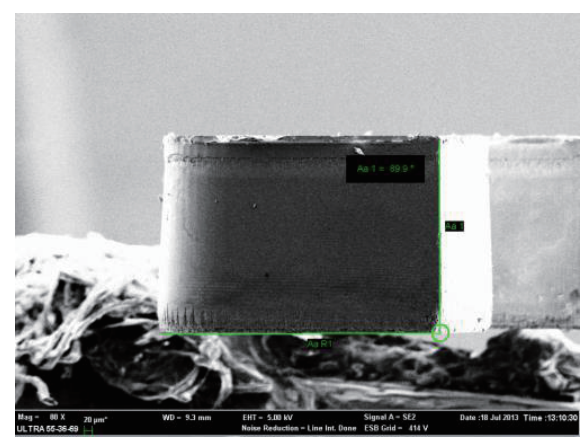

(c)

Fig. 8. (Color online) Fabricated cooling arm. (a) Image of cooling arm. (b) SEM image of clamping arm. (c) Enlarged side view of the rectangle area. 


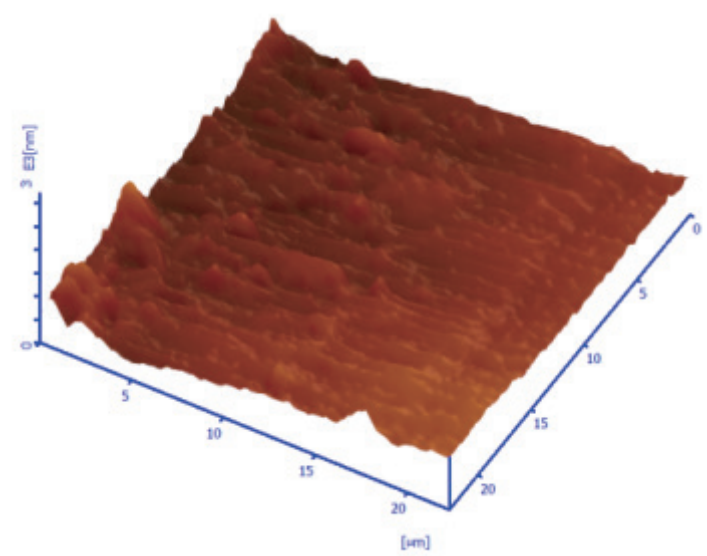

(a)

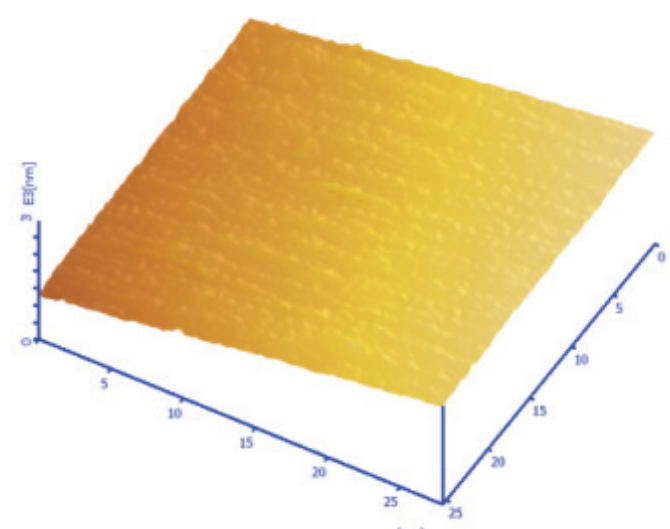

(b)

Fig. 9. (Color online) AFM image of DRIE surface. (a) Before KOH:IPA polishing. (b) After KOH:IPA polishing.

\section{Fracture Failure Test}

Because the cooling arms are used to clamp the TMP assembly, it is necessary to measure the deformation that the clamping arms can endure. A strength testing machine (RHESCA PTR-1101), as shown in Fig. 10(a), is used to measure the fracture force and deformation of the clamping arm. The test probe drives the clamping arm deformation in the radial direction. As can be seen from the graph, the relationship between the displacement and the force is approximately linear. When the deformation of the 

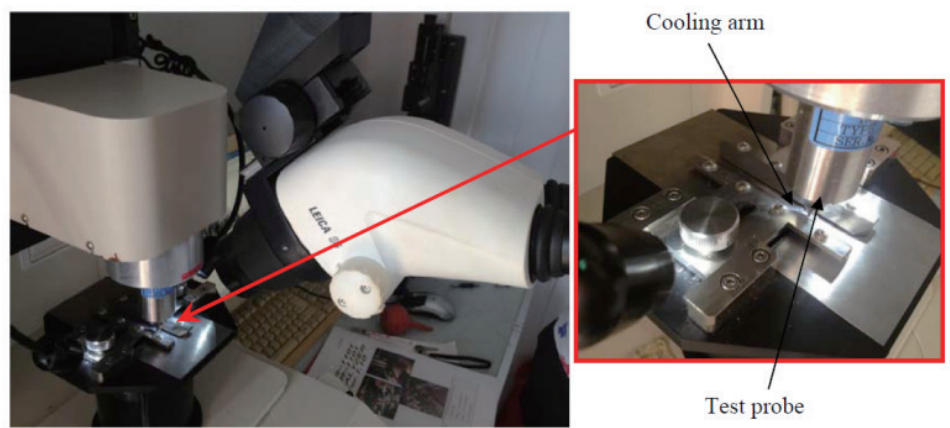

(a)

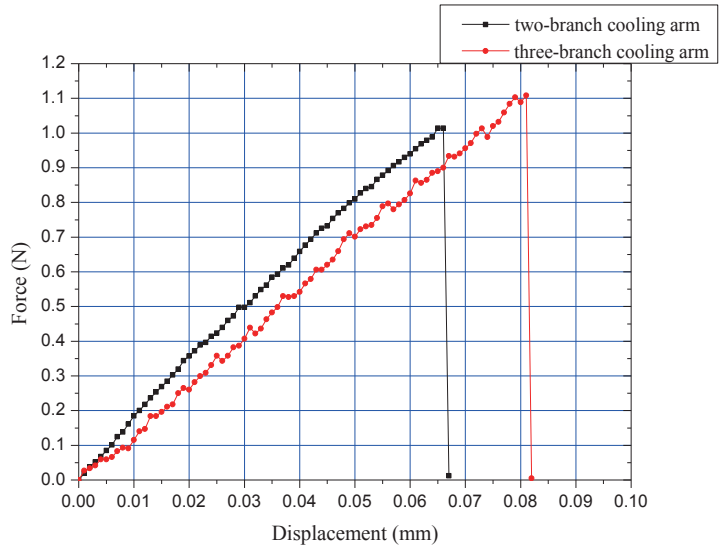

(b)

Fig. 10. (Color online) Mechanical test.

(a) Strength testing platform.

(b) Curve of the displacement vs force.

clamping arm is $20 \mu \mathrm{m}$, the grip forces of the two-branch and three-branch cooling arms are 0.26 and $0.357 \mathrm{~N}$, respectively. The corresponding maximum stresses of the twobranch and three-branch cooling arms are 0.395 and $0.574 \mathrm{GPa}$, respectively, which are consistent with the simulation results. The fracture deformations of the two-branch and three-branch cooling arms are 0.64 and $0.82 \mu \mathrm{m}$, respectively. Thus, the three-branch cooling arm can endure a larger deformation than the two-branch cooling arm. In other words, the three-branch cooling arm has a larger mechanical strength safety coefficient than the two-branch cooling arm. 


\section{Conclusions}

On the basis of theoretical analysis and finite element simulation, the three-branch cooling arm was found to have a smaller temperature difference on the clamping surface and better strength than the cooling arm with fewer branches. The mechanical strength test result is consistent with the simulation result. The three-branch cooling arm can endure a $0.82 \mu \mathrm{m}$ deformation in the radial direction, whereas the two-branch cooling arm can endure $0.64 \mu \mathrm{m}$. As can be seen from the AFM test, the smoothness of the clamping surface can be improved by KOH:IPA polishing.

\section{Acknowledgements}

This work was supported in part by the Shanghai Municipal Science and Technology Commission (Nos. 11JC1405700 and 13511500200), SRFDP (No. 20130073110087), the National Natural Science Foundation of China (Nos. 61176104 and 51475307), 973 Program (No. 2013CB329401), and NDPR Foundation of China (No. 9140A26060313JW3385).

\section{References}

1 J. D. Lindl: Phys. Plasmas 2 (1995) 3933.

2 J. Zhang: Physics 3 (1999) 142.

3 J. D. Lindl, R. L. McCrory and E. M. Campbell: Phys. Today 45 (1992) 32.

4 C. Castro, R. C. Montesanti, J. S. Taylor, A. V. Hamza and E. G. Dzenitis: ASPE 24th Annual Meeting, Monterey, CA, United States, 2009.

5 E. T. Alger, J. Kroll, E. G. Dzenitiz, R. Montesanti, J. Hughes, M. Swisher, J. Taylor, K. Segraves, D. M. Lord, J. Reynolds, C. Castro and G. Edwards: Fusion Sci. Technol. 59 (2011) 78.

6 R. Miles, J. Hamilton, J. Crawford, S. Ratti, J. Trevino, T. Graff, C. Stockton and C. Harvey: Fusion Sci. Technol. 55 (2009) 308.

7 H. Khater and S. Brereton: Fusion Engineering, SOFE 2009, 23rd IEEE/NPSS Symposium, San Diego, CA, United States, 2009.

8 C. Montesant, M. Seugling, L. Klingman, G. Dzenitis, T. Alger, L. Miller, A. Kent, C. Castro, L. Reynolds and A. Carrillo: American Society for Precision Engineering 23rd Annual Meeting, Portland, OR, United States, 2008.

9 M. Q. Huda, T. M. F. Amin, Y. Ning, G. McKinnon, J. Tulip and W. Jager: Conference on Micromachining and Microfabrication Process Technology XVII, San Francisco, United States, 2012.

10 M. Q. Huda, T. M. F. Amin, Y. Ning, G. McKinnon, J. Tulip and W. Jager: Conference on Micromachining and Microfabrication Process Technology XVIII, San Francisco, United States, 2013.

11 R. Agarwal, S. Samson and S. Bhansali: J. Micromech. Microeng. 13 (2007) 26.

12 I. Zubel, K. Rola and M. Kramkowska: Sens. Actuators, A 171 (2011) 436.

13 D. Nilsson, S. Jensen and A. Menon: J. Micromech. Microeng. 13 (2003) 57.

14 D. Lee, K. Yu, U. Krishnamoorthy and O. Solgaard: Microelectromech. Syst. 18 (2009) 217. 